\title{
Modelling the effect of aerosol polydispersity on unipolar charging and measurement in low-cost sensors
}

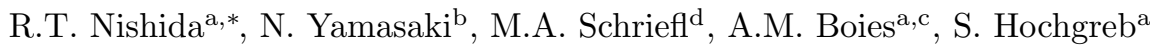 \\ ${ }^{a}$ Department of Engineering, University of Cambridge, Cambridge CB2 1PZ, UK \\ ${ }^{b}$ Department of Chemical Engineering and Biotechnology, University of Cambridge, Cambridge CB3 OAS, UK \\ ${ }^{c}$ Department of Civil, Environmental and Geo-Engineering, University of Minnesota, Minneapolis, Minnesota 55455, USA \\ ${ }^{d}$ Institute of Electronic Sensor Systems, Graz University of Technology, Graz A-8010, Austria
}

\begin{abstract}
Low-cost ultrafine aerosol sensors are experimentally calibrated with controlled aerosol sources to provide metrics such as surface area, lung-deposited surface area, mean particle size and/or total concentration from one or more electrical current measurements. However, an aerosol with a large standard deviation in particle size provides a significantly different signal from a monodisperse aerosol with the same median particle size. In this paper, we investigate the effect of particle polydispersity on measurements in devices which employ unipolar charging. The conservation equations are solved for particle/ion charging and transport (convection, diffusion and electrical transport) in laminar, steady-state, incompressible flow. Lognormal particle size distributions are represented by over $10^{2}$ coupled conservation equations for multiple size bins and discrete charge states and solved numerically for the first time. Modelling results show that integrated electrical current from a polydisperse particle distribution can be represented by a monodisperse distribution characterised by the count mean diameter ( $\bar{d}$; unipolar diffusion charging) or diameter of the average surface $\left(d_{\bar{s}}\right.$; photoelectric charging) and total concentration, for a large range of particle distributions and operating conditions offering a convenient simplification for the interpretation of ultrafine particle measurements. The simplification reduces the number of simultaneous conservation equations required, thereby reducing computation time by up to 57 times for a polydisperse particle distribution represented by 16 discrete size bins. The method of analysis is useful to both users and developers of low-cost ultrafine particle sensors to understand the effect of particle polydispersity on measurements.
\end{abstract}

\section{Introduction}

Compact, low-cost sensors for field measurements of ultrafine particles combine charging, classification and detection into smaller, sometimes handheld, devices. The devices each include a charging stage in which aerosol particles acquire discrete charge states. An ion trap captures highly mobile, excess ions with an electric field and the remaining charged particles induce electrical currents within sensitive electrometers. These devices provide a low-cost method of measuring ultrafine particles for environmental or personal exposure monitoring and sufficient understanding of the charging and transport effects is required for interpretation of measurements.

Several low-cost sensors use a corona discharge process to generate unipolar ions, which subsequently transfer charge to particles via diffusion charging. The integrated electrical current resulting from unipolar diffusion charging is linearly dependent on number concentration and nearly linearly dependent on particle diameter for monodisperse particles: $i \sim N d$ [22] for the NanoTracer, $i \sim N d^{1.13}$ for the NSAM [15], and $i \sim N d^{1.1}[2,10,29]$ for the Naneos Partector and DiSCmini.

*Corresponding author. E-mail address: rn359@cam.ac.uk
The NanoTracer and DiSCmini give an additional estimate of mean size and number concentration. The NanoTracer operates a low-efficiency electrostatic precipitator which alternately captures more electrically mobile particles with a square wave voltage, yielding two signals [1]. In the DiSCmini, there are two consecutive filter stages in which particles are preferentially deposited based on size. In both devices, two signals are used to derive mean particle diameter and concentration, assuming a standard deviation of a lognormal distribution and may give concentration estimates with accuracy of order $\pm 30 \%[10,16]$.

Alternative low-cost photoelectric charging devices ionize particles directly with ultraviolet (UV) light rather than by diffusion charging. Ions emitted during the photoionization process are captured in an ion trap and the remaining positively charged particles are collected on a filter inside a Faraday cup electrometer. The electrical current measurements are linearly proportional to photoelectrically active area and total (mobility) surface area $i \sim N d^{2}$ using the Alphasense UPM-S1 [26] and similar photoelectric charging methods and aerosol sensors $[5,6,19,24,32]$.

Low-cost ultrafine aerosol sensors are experimentally calibrated with monodisperse aerosol sources. However, they are exposed to polydisperse particle distributions dur- 
ing in-field operation. Fierz et al. [10] investigated the response of the Diffusion Size Classifier (DiSC) to a lognormal, polydisperse aerosol input. They found that a variation in geometric standard deviation $\left(1.1<\sigma_{g}<2.9\right)$ of the input aerosol resulted in significant prediction errors for diameter (up to 65\%) and concentration (up to 20\%) when assuming $\sigma_{g}=1.7$.

In this work, we quantify the effect of aerosol particle polydispersity on electrical currents resulting from unipolar diffusion and photoelectric charging mechanisms in low-cost ultrafine particle measurement devices. Numerical models of particle charging and transport are developed to solve the conservation equations for multiple particle size bins and charge levels. A model for charging of polydisperse particle distributions is constructed which considers the coupling between ion concentration and particle concentration from each of the discrete size bins which make up the polydisperse distribution. The results are compared with those from a model assuming a monodisperse particle distribution at a representative particle diameter. The numerical simulations are used to demonstrate the effect of particle polydispersity on measurements from low-cost aerosol sensors for a range of input particle size distributions and operating conditions.

\section{Theory}

\subsection{Unipolar aerosol charging}

Unipolar diffusion charging and photoelectric charging both involve charge transfer between ions and particles. In unipolar diffusion charging, ions generated from a unipolar source such as corona discharge transfer charge to particles. The collision coefficient, $\beta$, quantifies the charge transfer rate between charged particles and ions and is applied using the method of Hoppel and Frick [13] and Fuchs [11] which includes electrostatic and diffusive forces.

$$
\beta=\frac{\pi \delta^{2} c \alpha_{C}(q)}{\exp \left(\frac{\varphi(i(q), \delta)}{k_{B} T}\right)+\frac{\delta c \alpha_{C}(q)}{4 D_{\mathrm{i}}} \int_{0}^{1} \exp \left(\frac{\varphi(i(q), \delta / x)}{k_{B} T}\right) \mathrm{d} x}
$$

where

$\varphi(i, r)=V_{e}\left(\frac{i}{r}-K \frac{(d / 2)^{3}}{2 r^{2}\left(r^{2}-(d / 2)^{2}\right)}\right)$.

The collision rate equation (1) includes the mean molecular velocity, $c$, and collisional cross sectional area, $\pi \delta^{2}$, where $\delta$ is the limiting sphere radius. The collision rate also depends on ion diffusivity, $D_{i}$, Boltzmann's constant, $k_{B}$, and temperature, $T$. The ion-particle collision probability, $\alpha_{C}$, depends on charge and distance [27]. The electrostatic potential, $\varphi$, is a function of particle material, diameter, $d$, and distance from the center of the particle, $r$, where $K=1$ for a perfectly conducting particle. The number and polarity of particle charges, $i$, is positive if the ion and particle are of the same polarity. Collision of an ion with a charged particle assumes the ion transfers charge to the particle, leading to a sink term in the conservation equations for both particles and ions.

During photoelectric charging, photon energy is absorbed by particles, causing the emission of electrons, which in turn collide with neutral gases, forming gaseous ions. For each emitted electron, the remaining particle retains a positive charge. The photoionization rate, $\alpha$, of a particle increasing from charge level $q$ to $q+1$ follows the equation

$$
\alpha^{q \rightarrow q+1}=K_{c}\left(h \nu-\Phi^{q \rightarrow q+1}\right)^{m} \frac{I \sigma_{\mathrm{ph}}}{4 h \nu} .
$$

where the proportionality constant, $K_{c}$, exponent, $m$, and work function, $\Phi$, are material dependent [5, 14]. Photoemission occurs while the product of the wavelength of light, $\nu$, and Planck's constant, $h=6.626068 \times 10^{-34} \mathrm{~m}^{2} \mathrm{~kg} / \mathrm{s}$, is higher than the work function, $\Phi$, of the particle. The work function increases with each emitted electron due to the increase in electrostatic attraction between the particle and the emitted charge. The emission rate is linearly proportional to the photoelectrically active area, $\sigma_{\mathrm{ph}}$, as well as the particle (mobility) surface area for a given particle material and morphology $[5,6,18,19,24,26,28,32]$. It is assumed that each photoemitted electron in turn forms a negatively charged gaseous ion. Ions generated via photoemission may recombine with the remaining, oppositely charged particles according to Eq. 1, thereby reducing the net photoelectric effect. More detail on the photoelectric charging and ion-particle collision equations can be found in Nishida et al. [25, 26].

\subsection{Aerosol polydispersity}

Aerosols are characterised by different geometric parameters and the method of characterisation depends on which quantities are measurable. Even controlled aerosols come in a range of particle sizes, and it is intractable to measure geometric properties of each particle individually. It is thus often convenient to describe the mean geometric properties by the $p$-th moments in its marginal size probability distribution $p(d)$,

$$
\begin{aligned}
\left(d_{\bar{p}}\right)^{p} & =\int_{0}^{\infty} p(d) d^{p} \mathrm{~d} d \\
\left(d_{\bar{p})^{p}}\right. & \approx \frac{\sum^{b}\left(N_{b} d_{b}^{p}\right)}{N} \\
d_{\bar{p}} & \approx\left(\frac{\sum^{b}\left(N_{b} d_{b}^{p}\right)}{N}\right)^{1 / p}
\end{aligned}
$$

where $N_{b}$ is the concentration of particles at particle size $d_{b}, N$ is total concentration, and $p$ is the exponent, or moment, of diameter. A higher moment yields a higher moment average, $d_{\bar{p}}$. The count mean diameter, $\bar{d}=d_{\overline{1}}$, is 
found for $p=1$, and the diameter of the average surface, $d_{\bar{s}}=d_{\overline{2}}$, for $p=2$, using notation from Hinds [12].

A lognormal distribution of particle concentration as a function of size applies to most single source aerosols which are dominated by collisional growth. The lognormal distribution based on particle count is commonly characterised by a count median diameter, $\mathrm{CMD} \approx$ $\exp \left(\sum^{b}\left(N_{b} \ln d_{b}\right) / N\right)$, and geometric standard deviation, $\sigma_{g}$. The CMD of a lognormal count distribution is the geometric mean diameter and should not be confused with the (arithmetic) count mean diameter, $\bar{d}$, where by definition, $\mathrm{CMD}<\bar{d}<d_{\bar{s}}$. Figure 1 shows $d_{\bar{s}}$ for two aerosols with different $\sigma_{g}$, but the same CMD and total concentration. By characterizing an aerosol with only count median diameter and total concentration, the polydispersity is neglected. For a lognormal size distribution the $p$-th moment diameter can be reckoned from CMD and $\sigma_{g}$ using the Hatch-Choate equation [12]:

$$
d_{\bar{p}}=\mathrm{CMD} \exp \left(\frac{p}{2} \ln ^{2} \sigma_{g}\right)
$$

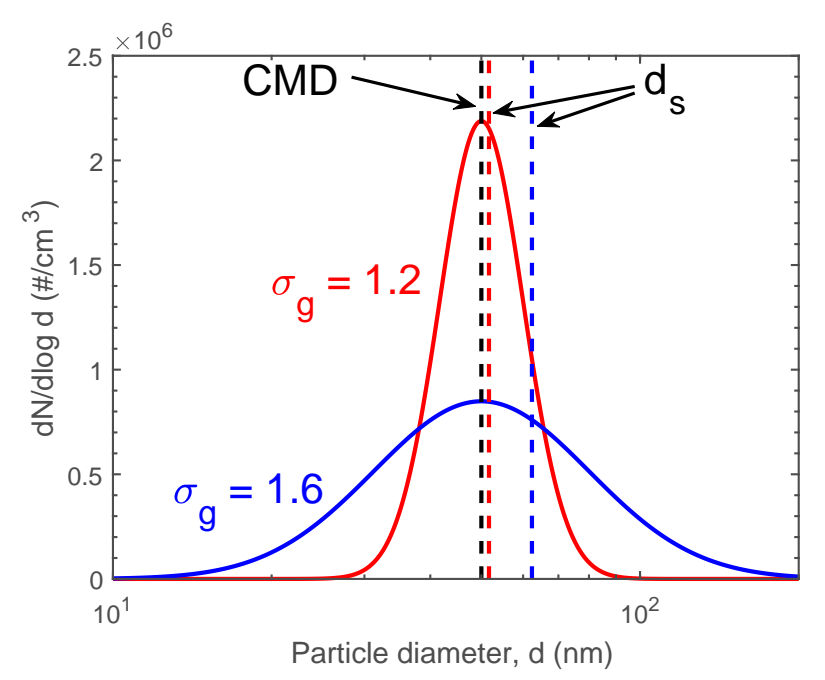

Figure 1: Two particle size distributions with the same CMD (50 $\mathrm{nm})$ and total concentration $\left(N=10^{6} \mathrm{~cm}^{-3}\right)$ with different standard deviations, $\sigma_{g}=1.2$ (red) and $\sigma_{g}=1.6$ (blue). The diameter of the average surface, $d_{\bar{s}}$ is shown for each distribution.

Calibration measurements made with monodisperse aerosol sources $[2,10,15,22,29]$ show that the electrical current measured from unipolar diffusion charging scales approximately with $i \sim N d^{1}$ and in the case of photoelectric charging, $i \sim N d^{2}$. The relations are grounded in theory, as demonstrated analytically for unipolar diffusion charging $[21,30]$ and photoelectric charging [25] of monodisperse particles subject to assumptions such as neglecting the effects of size dependent particle losses and secondary charging and transport processes.

The total electrical current obtained from a polydisperse particle distribution is composed of the integral sum of the contribution from each monodisperse particle size bin.
The induced current is proportional to the first and second moment determined using Eq. 4, for unipolar diffusion charging and photoelectric charging, respectively. For a polydisperse aerosol made up of a number of individual, monodisperse bins, $b$, of particle size, $d_{b}$, this results in

$$
i \sim \sum^{b}\left(N_{b} d_{b}^{p}\right)=N d_{\bar{p}}^{p}
$$

where the aerosol size is not necessarily lognormally distributed.

Given the complexity of charging and transport as a function of particle diameter and concentration, it is often not possible to demonstrate the effect of polydispersity on the total electrical current. Therefore, in this work, a numerical model is used to show that the moment average diameters can be used to simplify the charging and transport equations for a large range of conditions. Moment averages may be used to interpret low-cost ultrafine particle measurements rather than the commonly used count median diameter.

\section{Numerical methods}

The general steady-state conservation equations are solved considering diffusion charging, photoelectric charging, convection, diffusion and electric field transport for both charged particles and ions of charge +1 and -1 . The general equations are solved for both unipolar diffusion charging and photoelectric charging in this work, taking into account the appropriate source terms. The geometry, discretisation, boundary conditions, properties and solution method are described as follows.

\subsection{Unipolar diffusion charging governing equations}

A polydisperse distribution is represented by particle sizes within a number of $M$ monodisperse size bins. The simultaneous particle conservation equations are shown in Eq. $9 \mathrm{a}$ for the concentration of particles, $N_{b, q}$, at each charge level, $q$, and size bin, $b$. For unipolar charging, a positive polarity corona discharge is used, so that there is no significant source of negative ions. The conservation of positive ions is shown Eq. 9b. Importantly, the particle and ion conservation equations are coupled through the ion-particle collision source/sink term such that the concentrations of one affect the other. The transport of particles and ions is described by the local gas velocity, $\vec{u}$, in the convection term, particle diffusivity, $D$, in the diffusion term, and electrical mobility, $Z$, times electric field vector $\vec{E}$ representing the electrical transport term. 


$$
\begin{gathered}
\nabla \cdot\left(\vec{u} N_{b, q}\right)=\nabla \cdot\left(D_{b, q} \nabla N_{b, q}\right)+\nabla \cdot\left(Z_{b, q} \vec{E} N_{b, q}\right) \\
+\beta_{b}^{q-1 \rightarrow q} N_{b, q-1} n_{+1}-\beta_{b}^{q \rightarrow q+1} N_{b, q} n_{+1} \\
\nabla \cdot\left(\vec{u} n_{+1}\right)=\nabla \cdot\left(D_{\mathrm{i},+1} \nabla n_{+1}\right)+\nabla \cdot\left(Z_{\mathrm{i},+1} \vec{E} n_{+1}\right) \\
-\sum_{b=1}^{M} \sum_{q=q_{\min }}^{q_{\max }}\left(\beta_{b}^{q \rightarrow q+1} N_{b, q} n_{+1}\right)
\end{gathered}
$$

The total outlet current at the outlet boundary, $i_{\mathrm{o}}$, consists largely of the stagnated convective flux of particle charge, with contributions from both ions and particles. The contribution to $i_{\mathrm{o}}$ from particles is dominant:

$$
i_{\mathrm{o}}=\int_{A}\left(\sum_{b=1}^{M} \sum_{q=q_{\min }}^{q_{\max }} q e \vec{u} N_{b, q}\right) \cdot \mathrm{d} A
$$

where $e$ is the electron charge and $A$ is the area of the outlet. However, the electrical current at each boundary is calculated in its generalised form according to Eq. A.1. At the ion trap at which electrodes are aligned with the flow, the total electrode current, $i_{\mathrm{e}}$, consists of the diffusive flux of ion charge enhanced by electric field transport as shown in Eq. A.1. The current measured for unipolar diffusion charging at the ion trap electrodes, $i_{\mathrm{e}}$, is made up largely of the ion charges, which is determined by the rate of ion flux from the corona discharge process rather than any properties of the aerosol.

\subsection{Photocharging governing equations}

The simultaneous particle conservation equations are shown in Eq. 11a for the concentration of particles, $N_{b, q}$, at each charge level, $q$, and size bin, $b$. The particle and ion governing equations are coupled through the source/sink terms for photoionization and ion-particle collision such that the concentrations of each affect the other. Photoemission causes the formation of negative ions which affect the rate of ion-particle collision. There is no significant source of positive ions. The governing equation for negative ions is shown in Eq. $11 \mathrm{~b}$.

$$
\begin{gathered}
\nabla \cdot\left(\vec{u} N_{b, q}\right)=\nabla \cdot\left(D_{b, q} \nabla N_{b, q}\right)+\nabla \cdot\left(Z_{b, q} \vec{E} N_{b, q}\right) \\
+\alpha_{b}^{q-1 \rightarrow q} N_{b, q-1}-\alpha_{b}^{q \rightarrow q+1} N_{b, q} \\
+\beta_{b}^{q+1 \rightarrow q} N_{b, q+1} n_{-1}-\beta_{b}^{q \rightarrow q-1} N_{b, q} n_{-1} \\
\nabla \cdot\left(\vec{u} n_{-1}\right)=\nabla \cdot\left(D_{\mathrm{i},-1} \nabla n_{-1}\right)+\nabla \cdot\left(Z_{\mathrm{i},-1} \vec{E} n_{-1}\right) \\
+\sum_{b=1}^{M} \sum_{q=q_{\min }}^{q_{\max }}\left(\alpha_{b}^{q \rightarrow q+1} N_{b, q}-\beta_{b}^{q \rightarrow q-1} N_{b, q} n_{-1}\right)
\end{gathered}
$$

The total current measured at the outlet boundary, $i_{\mathrm{o}}$, is similarly described by Eq. 10. Unlike the diffusion charging case, the current measured for photoelectric charging at the ion trap electrodes, $i_{\mathrm{e}}$, is made up largely of the ion charges directly resulting from photoelectric emission, thereby giving measurements of the properties of the aerosol $[25,26]$.

\subsection{Geometry, mesh and boundary conditions}

\subsubsection{Diffusion charger}

The ion source for unipolar diffusion chargers is usually a corona discharge process. The method for transporting ions to a charging section in which particles are exposed to the ions varies between devices. The ions may be transported through a charging section by an alternating or constant electric field [4], or by convection or diffusion. In this study we consider the simple case of a charging channel wherein the particle flow is passed through an ion wind, created by electric field transport of ions perpendicular to the flow direction.

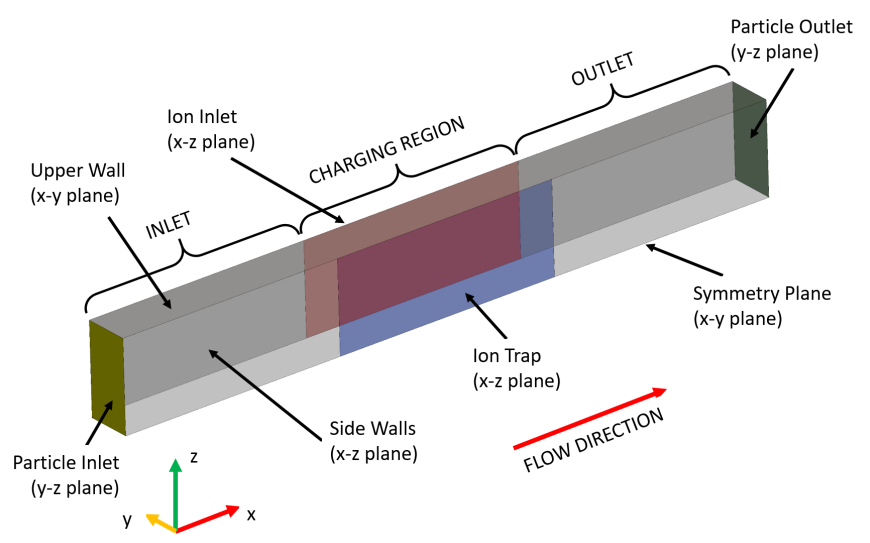

Figure 2: Geometry of the simulated diffusion charger. Boundaries are shown: Particle Inlet, Particle Outlet, Ion Inlet, Ion Trap, Side Walls, Upper Wall and Symmetry Plane. Boundary conditions for each boundary are found in Table B.4.

The geometry under consideration consists of a symmetrical rectangular flow channel with a length of $60 \mathrm{~mm}$ and a cross section of $4 \times 16 \mathrm{~mm}^{2}$ as shown in Fig. 2. The geometry is divided into three blocks: Inlet, Charging Region, and Outlet. Each block of the mesh consists of $70 \times 35 \times 70$ cells, yielding to a total cell number of 514,500 determined from a grid independence study. Simple grading with decreasing cell size towards the walls was applied in $y$ - and $z$-direction such that the cell size in the middle of the channel is four times the cell size at the walls.

The boundary conditions are listed in Table B.4. At the inlet boundary, neutrally charged, monodisperse particles enter the charger entrained in an air flow at a uniform prescribed velocity of $0.26 \mathrm{~m} \mathrm{~s}^{-1}$ corresponding to $1 \mathrm{~L}$ $\min ^{-1}$ at NTP. It is assumed that the ion generation region (corona discharge) is separated from the flow channel by a grounded grid which is transmissive for ions, denoted 
as Ion Inlet. The flow passes through the charging region between the Ion Inlet and Ion Trap boundaries in which the ions are transported and the diffusional charge transfer to the particles occurs. Positively charged ions are assumed to enter the geometry at the Ion Inlet at a potential of $0 \mathrm{~V}$, and are pulled towards the oppositely located Ion Trap, where a negative DC voltage is applied in order to capture excess ions. The charging efficiency of unipolar diffusion chargers is quantified by the $n t$ product (concentration of ions multiplied by time the aerosol is exposed to ions) which influences the charge states of the aerosol. Along with the collision rate, $\beta$, the $n t$ product makes up the ion-particle collision term of Eq. $9 \mathrm{~b}$ after integration. It is the main charging parameter by which diffusion charging instruments can be compared [7]. The ion inlet concentration (set at the Ion Inlet boundary) is chosen to match an averaged $n t$ product found by multiplying the ion concentration $n$ by the volume of the charging region $(20 \times 4 \times 16 \mathrm{~mm})$ and dividing the quantity by the volumetric flow rate. A precondition for this calculation is that the ions are confined within the charging region and that the ion concentration remains approximately constant throughout the charging region, a condition which is met for dominant electric field transport, i.e. the convective, diffusive, and ion-particle collision terms of Eq. 9b are negligible. This condition is ensured for all ion concentrations by setting the ion trap voltage to $-150 \mathrm{~V}$ such that nearly all the highly mobile ions are captured at the ion trap and are not significantly captured or lost elsewhere in the system.

It is assumed that the local charge density has a negligible effect on the local electric field in all cases. This assumption is valid for disperse particle and ion concentrations [17]. Biskos et al. [3] found the effect of net ionic charge to be significant for ion concentrations greater than $5 \times 10^{13}$ ions $\mathrm{m}^{-3}$. Local charge density could affect local electric fields significantly over the externally imposed electric field could potentially affect the transport of charged particles and ions. The net intrinsic and extrinsic particle charging efficiencies could be affected, therefore the effect of local charge density on the local electric field should considered in unipolar charger design. Analyses of these effects are outside of the scope of this work.

\subsubsection{Photoelectric charger}

In a direct ultraviolet (UV) photoelectric charger, particles are exposed directly to UV light and ions which result from that process are captured in an ion trap leaving charged particles remaining in the flow $[25,26]$. The photoelectric charger geometry considered in this work represents a that of a typical photoelectric charger and is not intended for direct comparison with diffusion charging devices. The geometry consists of a cylindrical flow chamber containing two concentric electrodes used for electric field transport of ions and particles. The outer electrode is a cylinder of $25 \mathrm{~mm}$ diameter and $200 \mathrm{~mm}$ length. The inner electrode is a concentrically located rod of $1.5 \mathrm{~mm}$ diame- ter. The charger volume is irradiated with ultraviolet light to ionize the particles. A bias voltage between the two electrodes provides an electric field within the photoionization region which captures highly mobile ions generated during photoionization. Charged particles are captured less readily due to their lower mobility $[25,26]$. The product of light intensity, $I$, and empirical constant, $K_{c}$, of Eq. 3 are fit to correspond with empirical data and are summarised in Table 1 along with the remaining operating conditions. A UV light source is placed at the upstream end of the system at the particle inlet and light is directed towards the outlet of the chamber. The non-collimated light intensity decreases with the inverse square law in the flow direction and the product $K_{c} I$ is fixed at the inlet, $2 \mathrm{~cm}$ from the light source. The particle work function, $\Phi_{\infty}$, for carbon particles is extracted from Michaelson [23].

The computational mesh comprises a $45^{\circ}$ section of the tube due to the axisymmetric nature of the solution. The mesh consists of six blocks of $10 \times 20 \times 50$ cells making up a total of $6 \times 10^{4}$ cells determined from a grid independence study. Simple grading towards the walls was applied where the highest concentration gradients occur. Detailed descriptions of the photoelectric charger geometry and computational mesh may be found in Nishida et al. [25, 26] along with numerical verification of the monodisperse particle charging equations.

The boundary conditions are summarised in Table B.5. At the electrodes, capture of particles and ions is due to diffusional and electric field transport of particles and ions. The concentration boundary conditions at the electrodes are set to zero for both particles and ions. It is assumed that the particles and ions which contact the walls are immediately captured and neutralised such that the charged particle and ion concentrations at the walls are equal to zero.

\subsection{Properties and Operating Conditions}

The particle flow comprises initially neutral charged particles in air at NTP conditions for both the diffusion and photoelectric charger. In each case, the velocity field is calculated using the laminar, steady-state, and incompressible form of the Navier-Stokes equations. The flow field solution is adopted as the advective term of the particle and ion concentration equations. Constant air density, viscosity, and negligible electro-hydrodynamic effects are assumed. The particle diffusion coefficient, $D_{b, q}$, and electrical mobility, $Z_{b, q}$, are functions of particle diameter calculated using mechanical mobility and Cunningham slip correction factors for air at NTP as described in Kulkarni et al. [20]. Collision rates, $\beta$, are generated using ion properties from Eq. 1 and Wiedensohler et al. [31]. A summary of the properties and operating conditions is given in Table 1.

\subsection{Solution Method}

The governing equations are solved using the CFD package OpenFOAM v.5. The three-dimensional, steady state, 
Table 1: Diffusion charger (DC) and photoelectric charger (PC) model parameters and operating conditions

\begin{tabular}{lllll}
\hline \hline Parameter & Symbol & Units & DC & PC \\
\hline Negative ion diffusivity & $D_{-1}$ & $\mathrm{~m}^{2} \mathrm{~s}^{-1}$ & - & $4.3 \times 10^{-6}$ \\
Positive ion diffusivity & $D_{+1}$ & $\mathrm{~m}^{2} \mathrm{~s}^{-1}$ & $3.41 \times 10^{-6}$ & - \\
Ion electrical mobility & $Z_{-1}$ & $\mathrm{~m}^{2} \mathrm{~V}^{-1} \mathrm{~s}^{-1}$ & - & $1.7 \times 10^{-4}$ \\
Ion electrical mobility & $Z_{+1}$ & $\mathrm{~m}^{2} \mathrm{~V}^{-1} \mathrm{~s}^{-1}$ & $1.35 \times 10^{-4}$ & - \\
Flow rate & $Q$ & $\mathrm{std} \mathrm{L} \mathrm{min}^{-1}$ & 1 & 1.5 \\
Bulk velocity & $U$ & $\mathrm{~ms}^{-1}$ & 0.2604 & $5.17 \times 10^{-2}$ \\
Residence time & $t$ & $\mathrm{~s}$ & 0.23 & 3.86 \\
Res. time, charging region & $t_{\mathrm{ch}}$ & $\mathrm{s}$ & $7.7 \times 10^{-2}$ & 3.86 \\
Empirical constant & $m$ & & - & 2 \\
Light energy & $h v$ & $\mathrm{eV}(\mathrm{nm})$ & - & $6.69(185)$ \\
Work function & $\Phi_{\infty}$ & $\mathrm{eV}$ & - & 4.95 \\
\hline \hline
\end{tabular}

conservation equations were discretized with Gaussian Finite Volume integration using linear or upwind (for advection divergence) numerical schemes. The resulting linear system of equations is solved using a Preconditioned Biconjugate Gradient (PBiCG) solver with a diagonal incomplete LU decomposition (DILU) preconditioner for asymmetric matrices (e.g. ion and particle concentrations, velocities) and Preconditioned Conjugate Gradient (PCG) solver with Diagonal Incomplete-Cholesky (DIC) preconditioners for symmetric matrices (pressure, potential). In each case, the initial conditions are set to zero for concentrations of ions and particles at all particle charge states and size bins throughout the computational mesh. The same two convergence criteria were used for each case, i.e. that the particles and charge generated within the volume equals the integral sum of the particle and charge flux at all boundaries to within $1 \times 10^{-3}$ particles per second and 0.1 aA, respectively.

The minimum charge level is set to -5 charges per particle such that the concentrations of all neutral and negatively charged particles are calculated. The maximum charge state is selected for each case as sufficiently high to ensure the concentration of particles at the highest charge state is negligible. In this work, the maximum charge state is set to +38 giving a total of 44 charge states. Solution time is a function the number of simultaneous equations to be solved, which is determined by the range of charge states and the number of particle size bins ranging from approximately one minute for monodisperse simulations to one hour for polydisperse simulations with 16 particle size bins. All calculations are performed using a desktop computer (Intel $\mathrm{R}$ ) Core $^{\mathrm{TM}}$ i7 $3.40 \mathrm{GHz}$ ) with $16 \mathrm{~GB}$ of RAM running on openSUSE 13.1 .

\subsection{Parameter variation}

Results from polydisperse particle size distributions represented by multiple size bins are compared with those using a single monodisperse size bin at a corresponding mean particle size and total concentration for each of the diffusion charging and photoelectric charging systems. Electrical current at the outlet, $i_{\mathrm{o}}$, relevant to low-cost measure- ment devices is compared for parameters varied through a range of aerosol properties expected in-field: standard deviation, $\sigma_{g}$, count median diameter, CMD, and total particle concentration, $N$. The amount of charging is varied using the $n t$ product for diffusion charging and the empirical constant, $K_{c} I$, for photoelectric charging. The parameter variation includes a set of baseline conditions around which each parameter is varied individually. Sixteen simulations are performed for each of the two chargers at each of three size distribution resolutions $(1,8$, and 16 bins) for a total of 96 simulations. Table 2 shows the range of input values varied either linearly or logarithmically throughout the range for each parameter. The two simulation conditions with the highest $n t$ products in the diffusion charging case include ion concentrations greater than $5 \times 10^{13}$ ions $\mathrm{m}^{-3}$. For similar charging conditions, it is recommended that for ion concentrations above this threshold, electrostatic dispersion due to high net charge density should be considered [3]. In this work, the effect is neglected and results should be analysed in the context of the $n t$ product in which a proportionally lower ion concentration and longer residence time could reach an equal $n t$ product.

A monodisperse particle size used to represent each evaluated polydisperse distribution is calculated as the mean diameter, $\bar{d}$, or the diameter of the average surface, $d_{\bar{s}}$, using Eq. 7 for unipolar diffusion charging and photoelectric charging, respectively. The conditions presented here are restricted to lognormal particle size distributions assumed in each case which is generally representative of single-source aerosols, though the moment average diameters may be calculated using Eq. 4 for other polydisperse distributions.

\section{Results}

\subsection{Numerical comparison and computation times}

Numerical results for diffusion charging (DC) and photoelectric charging (PC) using the baseline aerosol lognormal distribution and operating conditions of Table 2 are shown in Fig. 3. The lognormal distribution is represented by a 
Table 2: Range and spacing of variable parameters for model representative of expected aerosol and operating conditions.

\begin{tabular}{|c|c|c|c|c|c|c|}
\hline Variable & Units & Baseline & Minimum & Maximum & Spacing & Datapoints \\
\hline \multicolumn{7}{|l|}{ Input Aerosol } \\
\hline Standard deviation, $\sigma_{g}$ & - & 1.7 & 1.1 & 1.9 & Linear & 5 \\
\hline Count median diameter, CMD & $\mathrm{nm}$ & 50 & 30 & 200 & Logarithmic & 5 \\
\hline Particle concentration, $N$ & $\mathrm{~cm}^{-3}$ & $10^{6}$ & $10^{4}$ & $10^{7}$ & Logarithmic & 4 \\
\hline \multicolumn{7}{|l|}{ Photoelectric charging } \\
\hline Photoemission constant, $K_{c} I$ & $\mathrm{~J}^{-1} \mathrm{~m}^{-2} \mathrm{~s}^{-1}$ & $1 \times 10^{34}$ & $1 \times 10^{33}$ & $1 \times 10^{35}$ & Logarithmic & 5 \\
\hline Diameter of the average surface, $d_{\bar{s}}$ & $\mathrm{~nm}$ & 66 & 40 & 265 & calculated & 5 \\
\hline \multicolumn{7}{|l|}{ Unipolar diffusion charging } \\
\hline$n t$ product & $\mathrm{cm}^{-3} \mathrm{~s}$ & $1 \times 10^{6}$ & $1 \times 10^{4}$ & $1 \times 10^{8}$ & Logarithmic & 5 \\
\hline Count mean diameter, $\bar{d}$ & $\mathrm{~nm}$ & 58 & 35 & 230 & calculated & 5 \\
\hline
\end{tabular}

monodisperse

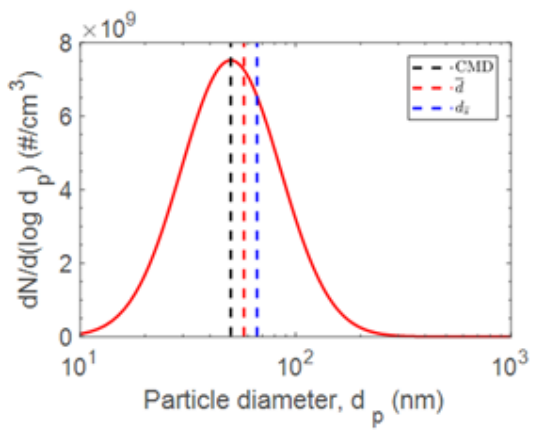

computation time: $502 \mathrm{~s}$

DC

PC

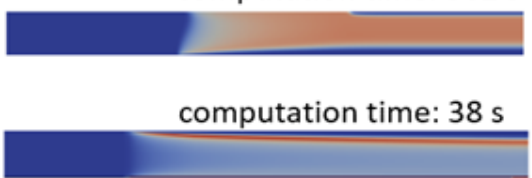

Flow direction
8 bins

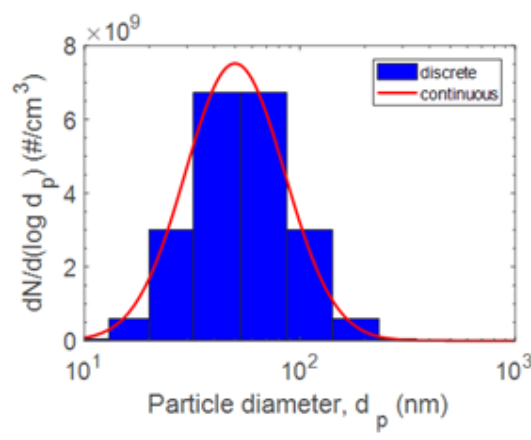

computation time: 2152 s

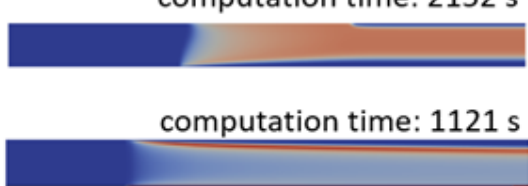

total charge: $\sum_{b} \sum_{q} q N_{b, q}\left[\frac{\#}{\mathrm{~m}^{3}}\right]$
16 bins

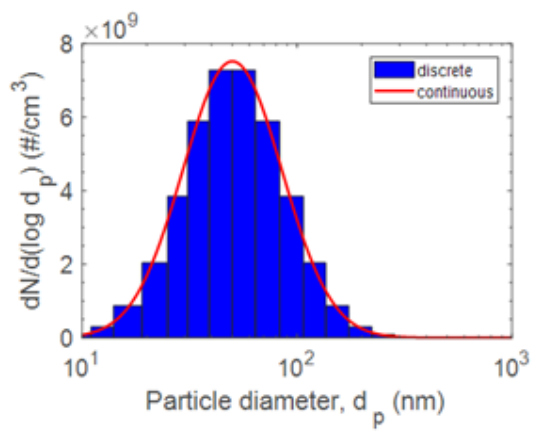

computation time: $8523 \mathrm{~s}$

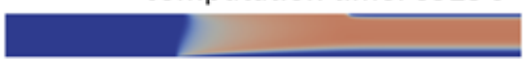

computation time: $1990 \mathrm{~s}$

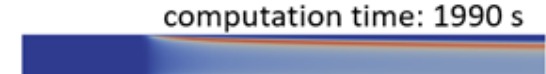

$5 e+10$

Figure 3: Lognormal particle size distribution represented by a single size bin at a representative mean diameter $(\bar{d}$; unipolar diffusion charging, DC, or $d_{\bar{s}}$; photoelectric charging, PC), 8 size bins, and 16 size bins for baseline operating conditions of Table 2 . The total charge, $\sum_{b} \sum_{q} N_{b, q} q$, is shown for a representative cross-section of the geometry for diffusion charging (x-z plane at $\mathrm{y}=2 \mathrm{~mm}$ ) and photoelectric charging (x-z plane at $\mathrm{y}=0 \mathrm{~mm}$ ) cases along with the total computation time required for each case. 
single, monodisperse size at a mean diameter $(\bar{d}$; unipolar diffusion charging or $d_{\bar{s}}$; photoelectric charging) or 8 or 16 size bins. Visualisations of the total charge throughout a representative cross-section of the geometry are shown to be equivalent for each calculation method for a given charger. For the diffusion charging baseline case, the calculation time for 8 bins and 16 bins is approximately 5 and 17 times that of the particle distribution represented by 1 bin, respectively. For the baseline case of photoelectric charging, the calculation time for 8 bins and 16 bins is approximately 29 and 52 times that of the particle distribution represented by 1 bin, respectively. Median and mean computation times for the 16 sets of operating conditions of Table 2 are shown in Table 3. Resolving the local charge states for a polydisperse distribution made up of multiple individual size bins significantly increases the computation time over a that of a single size bin. For the unipolar diffusion charging case, the calculation time for 8 bins and 16 bins is approximately 7 and 17 times that of the particle distribution represented by 1 bin, respectively. For the photoelectric charging case, the calculation time for 8 bins and 16 bins is approximately 31 and 57 times that of the particle distribution represented by 1 bin, respectively. The mean computation times for the photoelectric charging case are higher than the median times primarily to the relatively long computation times required for a few cases with the largest mean particle size (e.g. $200 \mathrm{~nm}$ ). The largest particle sizes hold the highest charge states, therefore the ion-particle recombination is relatively more significant due to the high electrostatic attraction of highly charged particles with ions. The effect of ion-particle recombination is more significant, involving increased coupling between the source terms of the ion and particle conservation equations.

The photoelectric charging cases with 8 or 16 size bins each require approximately five times more computation time per computational cell and equation solved than that of the single size bin due to the significant coupling between the population of ions generated during photocharging and the rate of ion-particle collisions in each size bin and charge state. For photoelectric charging, the ion concentration is strongly dependent on the particle charge distributions and vice versa. The unipolar diffusion cases with multiple size bins require a similar computational time per cell and per equation solved as that of the single size bin. The diffusion charging case has no volumetric ion source and the concentration of ions is not significantly affected by the ion-particle collisions for the present conditions. Therefore, there is little coupling of charging effects across individual particle size bins or charge states. The ion concentration may therefore be considered independent of the particle concentration, thereby simplifying the third term on the RHS of Eq. $9 \mathrm{~b}$.

\subsection{Count Mean Diameter vs. Count Median Diameter}

Simulations were performed for polydisperse size distributions represented by a moment average diameter, count

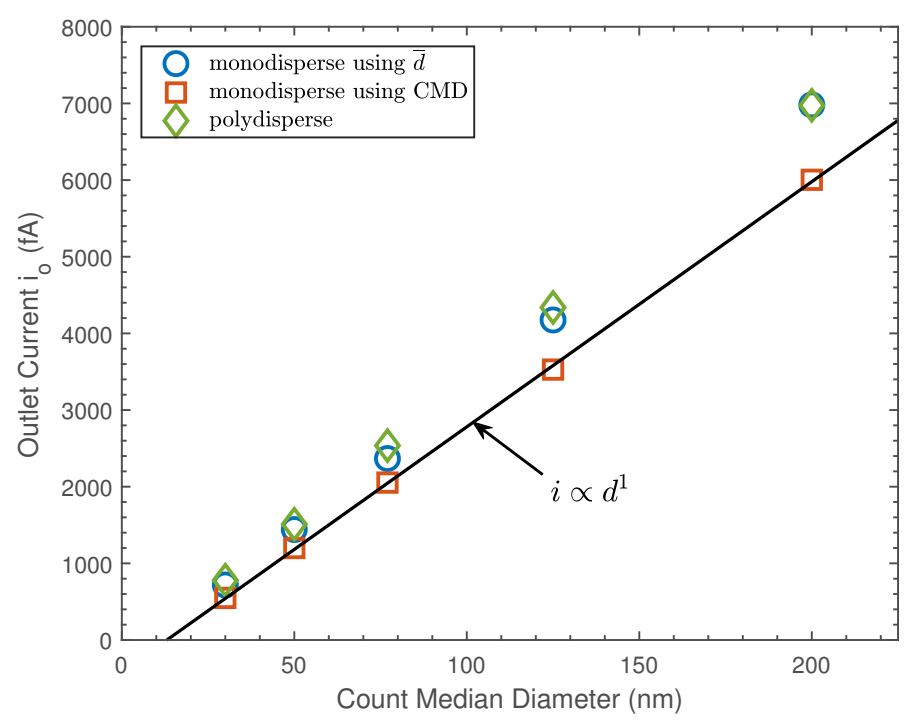

(a) unipolar diffusion charger

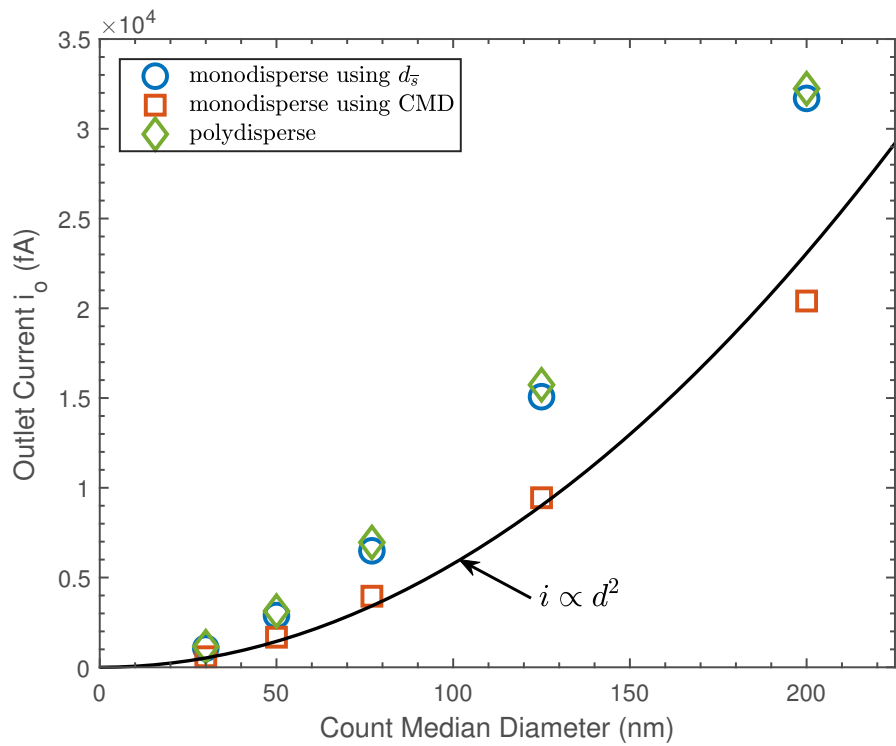

(b) photoelectric charger

Figure 4: Outlet current, $i_{\mathrm{O}}$, as a function of the CMD for polydisperse simulation with $N_{b i n}=8$, in comparison with monodisperse results using CMD and representative diameter (a) $\bar{d}$; unipolar diffusion charger (b) $d_{\bar{s}}$; photoelectric charger at baseline conditions 
Table 3: Simulation time comparison for diffusion charger (DC) and photoelectric charger (PC). Simulation times are presented as the median and mean times for the 16 different operating conditions of Table 2.

\begin{tabular}{lccccccc}
\hline \hline $\begin{array}{l}\text { Polydisperse } \\
\text { size bins }\end{array}$ & $\begin{array}{c}\text { Simultaneous } \\
\text { Conc. Eq.'s }\end{array}$ & \multicolumn{3}{c}{ Computation time (DC) } & \multicolumn{3}{c}{ Computation time (PC) } \\
\hline 1 & 44 & 385 & 432 & $1 \times$ & 38 & 63 & $1 \times$ \\
8 & 352 & 2866 & 2916 & $7 \times$ & 1121 & 2645 & $31 \times$ \\
16 & 704 & 7173 & 7187 & $17 \times$ & 1990 & 5040 & $57 \times$ \\
\hline \hline
\end{tabular}

median diameter, and 8 particle size bins for 5 different count median diameters, logarithmically spaced (30, 50, 77,125 and $200 \mathrm{~nm}$ ). The count mean diameter was calculated according to Eq. 7 using the baseline conditions of Table 2. Results shown in Fig. 4 confirm the polydisperse distribution may be represented by the moment average diameter (Fig. 4a: $\bar{d}$; unipolar diffusion charging and Fig. 4b: $d_{\bar{s}}$; photoelectric charging) at the same total concentration to estimate electrical current, $i_{\mathrm{o}}$, whereas the CMD is not representative. Figure $4 \mathrm{a}$ shows that the electrical current from diffusion charging is nearly linearly proportional to the count median diameter. Similarly, Fig. 4b shows that the electrical current from the photoelectric charger is nearly proportional to CMD raised to the power of two. Using the CMD as a size input for the monodisperse simulation causes significant deviations from polydisperse results, showing an increasing underestimation of electrical current with particle size. The maximum relative error was found to be $26 \%$ and $45 \%$ for the diffusion charger and photoelectric charger, respectively. The error when using CMD as the size input increases with standard deviation $[10]$.

\subsection{Accuracy in output current}

Numerical results for diffusion and photoelectric chargers with input aerosol parameters and operating conditions of Table 2 are shown in Fig. 5. Electrical current at the outlet, $i_{\mathrm{o}}$, of each charger is shown for a monodisperse aerosol using the moment average diameter (Fig. 5a: $\bar{d}$; unipolar diffusion charging and Fig. 5b: $d_{\bar{s}}$; photoelectric charging) compared with $i_{\mathrm{o}}$ from the polydisperse distribution represented by 16 size bins. Simulations with 44 charge states requires 704 coupled charge conservation equations to be computed for polydisperse cases with 16 bins, whereas only 44 charge conservation equations are required for monodisperse distributions. For the range of conditions in Table 2, the maximum percent difference in outlet current between the particle size distribution represented by 16 size bins and the distribution represented by one moment average diameter is $3.4 \%$ and $4.8 \%$ for the diffusion charger and photoelectric charger cases, respectively. If a significant fraction of charged particles is captured by the electric field of the ion trap and/or by diffusional losses to walls, the current calculations based on moment averages may not agree with the detailed calculations of polydisperse distributions, since the capture is dependent on the particle mobility and charge states [26].

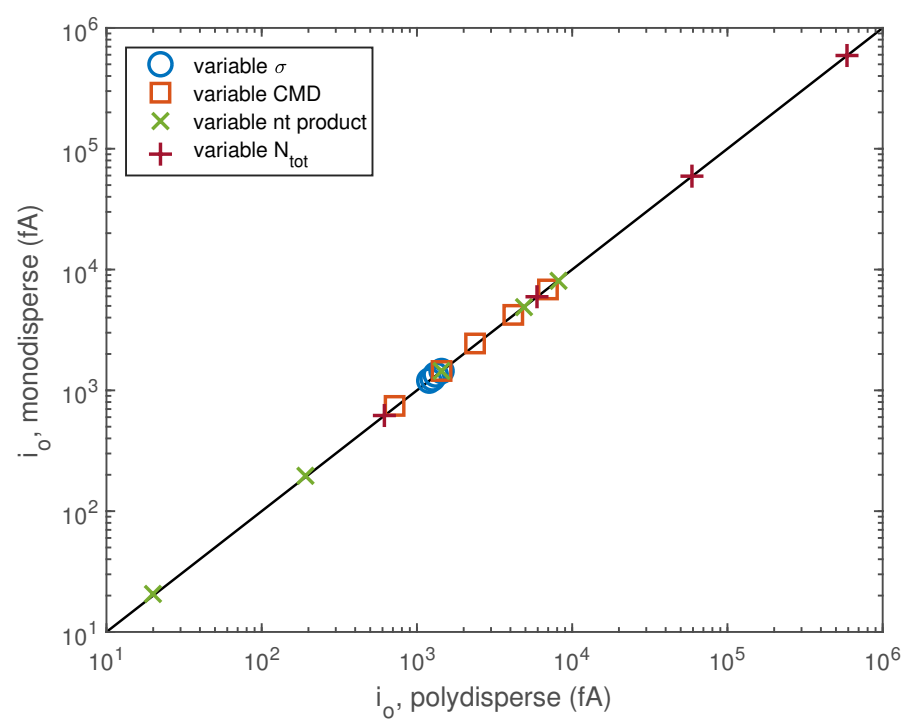

(a) unipolar diffusion charger

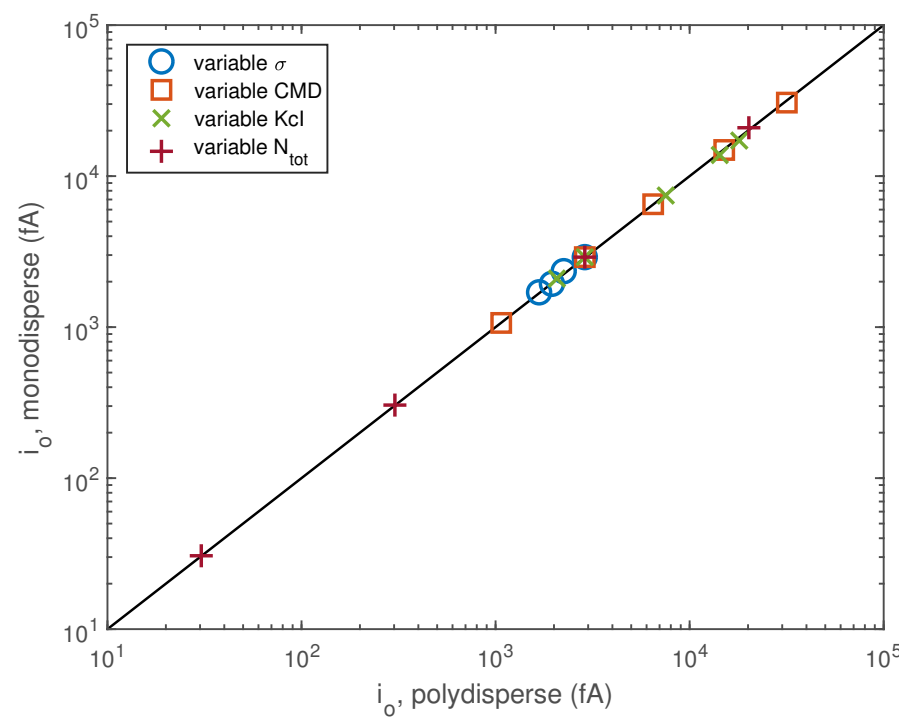

(b) photoelectric charger

Figure 5: Outlet current, $i_{\mathrm{o}}$, from monodisperse input at moment average diameter as a function of output current from polydisperse input with with $N_{b i n}=16$ for (a) $\bar{d}$; diffusion charger and (b) $d_{\bar{s}}$; photoelectric charger 
The operation of the NanoTracer and unipolar charging devices which employ low-efficiency eletrostatic precipitators rely on this principle to derive particle diameter and concentration estimates [1,22]. The effect of electric field strength on size dependent particle capture is discussed in the literature and is outside the scope of this work $[8,9,22,25,26]$. The range of particle size demonstrated in this work (30-200 nm CMD) is representative of those measured using low-cost ultrafine particle measurement devices whereas particles below $30 \mathrm{~nm}$ are less likely to charge or have a significant effect on the measured outlet currents.

\section{Conclusions}

In this work, the effect of aerosol particle polydispersity on electrical currents resulting from unipolar diffusion and photoelectric charging are analysed. A model for charging of polydisperse particle distributions is developed and solved numerically for the first time considering charge transfer between ions and particles from each of the discrete size bins which make up polydisperse distributions. The results are compared with those from a model assuming a monodisperse particle distribution with the same total concentration at either of the first two moments of a particle size distribution: the count mean diameter ( $\bar{d}$; unipolar diffusion charging) or the diameter of the average surface $\left(d_{\bar{s}}\right.$; photoelectric charging). For a representative range of input aerosol parameters and operating conditions, the monodisperse model (44 charge states and coupled charge conservation equations) accurately predicts the electrical current output from a polydisperse particle distribution (represented by 16 size bins $\times 44$ charge states $=704$ simultaneous, coupled charge conservation equations) to within $3.4 \%$ and $4.8 \%$ and an average of 17 and 57 times lower computation time for the diffusion charger and photoelectric charger cases, respectively. The moment average diameters offer a convenient simplification to the conservation equations for the analysis of unipolar diffusion charging or photoelectric charging. This work demonstrates the first two moments of a lognormal particle size distribution may be used to interpret ultrafine particle measurements based on unipolar charging. The method of analysis is useful to both users and developers of low-cost ultrafine particle sensors to understand the effect of particle polydispersity on measurements.

\section{Acknowledgements}

The authors gratefully acknowledge Alphasense Ltd., Cambridge Trust, Natural Sciences and Engineering Research Council (NSERC), and the UK Aerosol Society for financial support.

\section{References}

[1] Asbach, C., Kaminski, H., Von Barany, D., Kuhlbusch, T. A. J., Monz, C., Dziurowitz, N., Pelzer, J., Vossen, K., Berlin, K., Dietrich, S., et al. (2012). Comparability of portable nanoparticle exposure monitors. Annals of occupational hygiene, 56(5):606621 .

[2] Bau, S., Zimmermann, B., Payet, R., and Witschger, O. (2015). A laboratory study of the performance of the handheld diffusion size classifier (discmini) for various aerosols in the 15-400 nm range. Environmental Science: Processes $\&$ Impacts, 17(2):261269.

[3] Biskos, G., Reavell, K., and Collings, N. (2005a). Electrostatic characterisation of corona-wire aerosol chargers. Journal of Electrostatics, 63(1):69-82.

[4] Biskos, G., Reavell, K., and Collings, N. (2005b). Unipolar diffusion charging of aerosol particles in the transition regime. Journal of Aerosol Science, 36(2):247-265.

[5] Burtscher, H. (1992). Measurement and characteristics of combustion aerosols with special consideration of photoelectric charging and charging by flame ions. Journal of Aerosol Science, 23(6):549-595.

[6] Burtscher, H., Künzel, S., and Hüglin, C. (1998). Characterization of particles in combustion engine exhaust. Journal of Aerosol Science, 29(4):389-396.

[7] Büscher, P., Schmidt-Ott, A., and Wiedensohler, A. (1994). Performance of a unipolar square wave diffusion charger with variable nt-product. Journal of Aerosol Science, 25(4):651-663.

[8] Cao, L. N. Y., Chen, S.-C., Fissan, H., Asbach, C., and Pui, D. Y. H. (2017). Development of a geometric surface area monitor (gsam) for aerosol nanoparticles. Journal of Aerosol Science, 114:118-129.

[9] Cao, L. N. Y. and Pui, D. Y. H. (2018). A novel weighted sum method to measure particle geometric surface area in real-time. Journal of Aerosol Science, 117:11-23.

[10] Fierz, M., Houle, C., Steigmeier, P., and Burtscher, H. (2011). Design, calibration, and field performance of a miniature diffusion size classifier. Aerosol Science and Technology, 45(1):1-10.

[11] Fuchs, N. A. (1963). On the stationary charge distribution on aerosol particles in a bipolar ionic atmosphere. Geofisica pura e applicata, 56(1):185-193.

[12] Hinds, W. C. (1999). Aerosol technology: properties, behavior, and measurement of airborne particles, 2nd ed. John Wiley \& Sons.

[13] Hoppel, W. A. and Frick, G. M. (1986). Ionaerosol attachment coefficients and the steady-state charge distribution on aerosols in a bipolar ion environment. Aerosol Science and Technology, $5(1): 1-21$.

[14] Jiang, J., Hogan Jr, C. J., Chen, D.-R., and Biswas, P. (2007). Aerosol charging and capture in the nanoparticle size range (6-15 $\mathrm{nm}$ ) by direct photoionization and diffusion mechanisms. Journal of Applied Physics, 102(3):034904.

[15] Jung, H. and Kittelson, D. B. (2005). Characterization of aerosol surface instruments in transition regime. Aerosol Science and Technology, 39(9):902-911.

[16] Kaminski, H., Kuhlbusch, T. A., Rath, S., Götz, U., Sprenger, M., Wels, D., Polloczek, J., Bachmann, V., Dziurowitz, N., Kiesling, H.-J., et al. (2013). Comparability of mobility particle sizers and diffusion chargers. Journal of Aerosol Science, 57:156-178.

[17] Kasper, G. (1981). Electrostatic dispersion of homopolar charged aerosols. Journal of Colloid and Interface Science, 81(1):32-40.

[18] Keller, A., Fierz, M., Siegmann, K., Siegmann, H. C., and Filippov, A. (2001). Surface science with nanosized particles in a carrier gas. Journal of Vacuum Science $\&$ Technology A: Vacuum, Surfaces, and Films, 19(1):1-8.

[19] Kittelson, D., Watts, W., Savstrom, J., and Johnson, J. (2005). Influence of a catalytic stripper on the response of real time aerosol instruments to diesel exhaust aerosol. Journal of Aerosol Science, 36(9):1089-1107.

[20] Kulkarni, P., Baron, P. A., and Willeke, K. (2011). Aerosol mea- 
surement: principles, techniques, and applications. John Wiley \& Sons.

[21] Liu, B. Y. H. and Pui, D. Y. H. (1977). On unipolar diffusion charging of aerosols in the continuum regime. In Plenary and Invited Lectures, pages 151-158. Elsevier.

[22] Marra, J., Voetz, M., and Kiesling, H.-J. (2010). Monitor for detecting and assessing exposure to airborne nanoparticles. Journal of Nanoparticle Research, 12(1):21-37.

[23] Michaelson, H. B. (1977). The work function of the elements and its periodicity. Journal of applied physics, 48(11):4729-4733.

[24] Niessner, R. (1986). The chemical response of the photo-electric aerosol sensor (pas) to different aerosol systems. Journal of Aerosol Science, 17(4):705-714.

[25] Nishida, R. T., Boies, A. M., and Hochgreb, S. (2017). Modelling of direct ultraviolet photoionization and charge recombination of aerosol nanoparticles in continuous flow. Journal of Applied Physics, 121(2):023104.

[26] Nishida, R. T., Boies, A. M., and Hochgreb, S. (2018). Measuring ultrafine aerosols by direct photoionization and charge capture in continuous flow. Aerosol Science and Technology, 52(5):546556.

[27] Reischl, G. P., Mäkelä, J. M., Karch, R., and Necid, J. (1996). Bipolar charging of ultrafine particles in the size range below 10 nm. Journal of Aerosol Science, 27(6):931-949.

[28] Schmidt-Ott, A., Baltensperger, U., Gäggeler, H. W., and Jost, D. T. (1990). Scaling behaviour of physical parameters describing agglomerates. Journal of Aerosol Science, 21(6):711-717.

[29] Todea, A. M., Beckmann, S., Kaminski, H., and Asbach, C. (2015). Accuracy of electrical aerosol sensors measuring lung deposited surface area concentrations. Journal of Aerosol Science, 89:96-109.

[30] White, H. J. (1951). Particle charging in electrostatic precipitation. Transactions of the American Institute of Electrical Engineers, 70(2):1186-1191.

[31] Wiedensohler, A., Lütkemeier, E., Feldpausch, M., and Helsper, C. (1986). Investigation of the bipolar charge distribution at various gas conditions. Journal of Aerosol Science, 17(3):413-416.

[32] Zhou, L., You, R., Tan, J., and Zachariah, M. R. (2013). Wavelength-resolved uv photoelectric charging dynamics of nanoparticles: comparison of spheres and aggregates. Aerosol Science and Technology, 47(6):672-680.
Table B.4: Diffusion Charger (DC) boundary conditions

\begin{tabular}{|c|c|c|c|}
\hline Boundary & Momentum & Concentration & Voltage \\
\hline Inlet & $\begin{array}{l}\vec{u}=U \hat{e}_{x}[\mathrm{~m} / \mathrm{s}] \\
\frac{\partial p}{\partial n}=0\end{array}$ & $\begin{array}{l}N_{0}=N_{0, i n} \\
N_{q \neq 0}=0 \\
n_{+1}=0\end{array}$ & $\frac{\partial V}{\partial n}=0$ \\
\hline Outlet & $\begin{array}{l}\frac{\partial p}{\partial n}=0 \\
p=1.01325[\mathrm{bar}]\end{array}$ & $\begin{array}{l}\frac{\partial N_{q}}{\partial n}=0 \\
\frac{\partial n_{+1}}{\partial n}=0\end{array}$ & $\frac{\partial V}{\partial n}=0$ \\
\hline Ion Inlet & $\vec{u}=\overrightarrow{0}, \frac{\partial p}{\partial n}=0$ & $N_{q}=0$ & $V=V_{I}$ \\
\hline Ion Trap & $\vec{u}=\overrightarrow{0}, \frac{\partial p}{\partial n}=0$ & $\begin{array}{l}n_{+1}=n_{+1, i n} \\
\frac{\partial N_{q}}{\partial n}=0 \\
\frac{\partial n_{+1}}{}=0\end{array}$ & $V=V_{T}$ \\
\hline Side Walls & $\vec{u}=\overrightarrow{0}, \frac{\partial p}{\partial n}=0$ & $\begin{array}{l}N_{q}=0 \\
n_{+1}=0\end{array}$ & $V=0[\mathrm{~V}$ \\
\hline Upper Wall & $\vec{u}=\overrightarrow{0}, \frac{\partial p}{\partial n}=0$ & $\begin{array}{l}N_{q}=0 \\
n_{+1}=0\end{array}$ & $\frac{\partial V}{\partial n}=0$ \\
\hline Symmetry Plane & $\begin{array}{l}\frac{\partial u_{x}}{\partial z}=0, \frac{\partial p}{\partial n}=0 \\
\frac{\partial u_{y}}{\partial z}=0 \\
u_{z}=0\end{array}$ & $\begin{array}{l}\frac{\partial N_{q}}{\partial n}=0 \\
\frac{\partial n_{+1}}{\partial n}=0\end{array}$ & $\frac{\partial V}{\partial n}=0$ \\
\hline
\end{tabular}

\section{Appendix A. General Equation for Electrical Cur-} rent

Table B.5: Photoelectric Charger (PC) boundary conditions

$$
\begin{aligned}
& i=\int_{A} \sum_{b=1}^{M} \sum_{q=q_{\min }}^{q_{\max }}\left(q e \vec{u} N_{b, q}+q e D_{b, q} \nabla N_{b, q}+q e Z_{b, q} \vec{E} N_{b, q}\right) \cdot \mathrm{d} A \begin{array}{llll}
\text { Boundary } & \text { Momentum } & \text { Concentration } & \text { Voltage } \\
\text { Inlet } & \begin{array}{l}
\vec{u}=U \hat{e}_{x} \\
\partial p
\end{array} & N_{0}=N_{0, i n} & \frac{\partial V}{\partial n}=0
\end{array} \\
& -\int_{A}\left(e \vec{u} n_{-1}+e D_{\mathrm{i},-1} \nabla n_{-1}+e Z_{\mathrm{i},-1} \vec{E} n_{-1}\right) \cdot \mathrm{d} A \\
& +\int_{A}\left(e \vec{u} n_{+1}+e D_{\mathrm{i},+1} \nabla n_{+1}+e Z_{\mathrm{i},+1} \vec{E} n_{+1}\right) \\
& \begin{array}{clll}
\text { Outlet } & \frac{\partial p}{\partial n}=0 & \frac{\partial N_{q}}{\partial n}=0 & \frac{\partial V}{\partial n}=0 \\
\mathrm{~d} A & p=101325 \mathrm{~Pa} & \frac{\partial n_{-1}}{\partial n}=0 & \\
\text { Rod electrode } & \vec{u}=\overrightarrow{0}, \frac{\partial p}{\partial n}=0 & N_{q}=0 & V=V_{R} \\
& & n_{+1}=0 &
\end{array} \\
& \text { Appendix B. Boundary Conditions }
\end{aligned}
$$

Angular Symmetry cyclic cyclic cyclic

